\title{
External-field effects on the optical spectra of self-assembled InP quantum dots
}

\author{
Mitsuru Sugisaki, ${ }^{1,2, *}$ Hong-Wen Ren, ${ }^{1,3}$ Selvakumar V. Nair, ${ }^{1,2}$ Kenichi Nishi, ${ }^{1,4}$ \\ and Yasuaki Masumoto ${ }^{1,5}$ \\ ${ }^{1}$ Single Quantum Dot Project, ERATO, Japan Science and Technology Corporation, Japan \\ ${ }^{2}$ Centre for Advanced Nanotechnology, University of Toronto, Haultain Building, 170 College Street, Toronto, \\ Ontario M5S 3E3, Canada \\ ${ }^{3}$ Applied Optoelectronics Incorporation, 13111 Jess Pirtle Boulevard, Sugar Land, Texas 77478 \\ ${ }^{4}$ Photonic and Wireless Devices Research Labs., NEC Corporation, 34 Miyukigaoka, Tsukuba, Ibaraki 305-8501, Japan \\ ${ }^{5}$ Institute of Physics, University of Tsukuba, Tsukuba, Ibaraki 305-8571, Japan
}

(Received 8 May 2002; revised manuscript received 24 September 2002; published 12 December 2002)

\begin{abstract}
The effects of external electric and magnetic fields on InP self-assembled quantum dots (QDs) were investigated by means of single dot spectroscopy. By systematically changing a bias applied to the sample, successive energy shifts of the photoluminescence (PL) peaks from excitons and biexcitons due to the quantum confined Stark effect were clearly observed. The quadratic Stark coefficient was evaluated to be of the order of $10^{-31} \mathrm{Fcm}^{2}$. The energy separation of the PL peaks arising from the excitons and biexcitons changed with the applied electric field, reflecting a slight difference of the Stark coefficient between the exciton and biexciton states. The existence of permanent dipole moments was also revealed in both the exciton and biexciton states. The spatial separations between the electrons and holes along the growth direction in a QD were estimated to be $7 \AA$ for the exciton state, and $8 \AA$ for the biexciton state. Further, the diamagnetic shift and the Zeeman splitting of the exciton states were clearly observed in a magnetic field. It was found that diamagnetic coefficient gradually decreases on decreasing the QD size. A simple qualitative model can explain that this result is due to competition between quantum confinement and magnetic confinement.
\end{abstract}

DOI: 10.1103/PhysRevB.66.235309

PACS number(s): 78.67.Hc, 71.35.-y, 73.61.Ey, 75.75.+a

\section{INTRODUCTION}

Zero-dimensional (0D) semiconductor structures or quantum dots (QDs), which are often referred to as artificial atoms, have attracted considerable interest recently due to their potential for applications and fundamental physics interest. From the basic physics point of view, one of the very interesting topics is experimental measurements of the wave functions on the confined electrons and holes. While a direct mapping of the wave functions is rather difficult, quantities that directly relate to the extent and shape of the electron and hole densities can be obtained by optical measurements in the presence of external fields.

In almost all presently available QD samples, the photoluminescence (PL) energy separation between the exciton and biexciton states is much smaller than their macroscopic PL bandwidth caused by the fluctuations in their size and shape. In order to eliminate the ambiguity due to these sizeshape fluctuations, the observation of a single QD is very important. Since the QDs show very sharp PL lines reflecting the density of states of a OD system, it is expected that even very small changes of their electronic energies can be easily measured.

By observing single QDs, important information and interesting phenomena hidden behind the inhomogeneous distribution have been clarified, such as many carrier effects, ${ }^{1-3}$ charged excitons, ${ }^{4,5}$ strong optical anisotropy, ${ }^{6-8}$ fluorescence intermittency, ${ }^{9,10}$ and photon anti-bunching. ${ }^{11-13}$ In order to observe a signal from a single QD, it is important to reduce the number of QDs to be excited and probed. The QDs have to be well separated from each other in comparison with the spatial resolution of the detection microscope system. Among the various OD systems investigated so far, the QDs formed thorough the Stranski-Krastanow (S-K) growth mode are most suitable for the study of a single QD because the QDs can be fabricated in a single layer. In the present work, we employed InP S-K QDs to explore their optical properties in the external fields.

The optical properties of single QDs in external fields have been investigated theoretically and experimentally by several groups. For example, the effects of electric field in a wide variety of QD systems have been reported in Refs. 5 and 14-24. Since each electronic state (e.g., one exciton, biexciton, and their excited states) shows a different energy shift with respect to the applied electric field, ${ }^{16,21,22}$ detailed $\mu$-PL measurements are necessary to obtain information on the wave functions of these states. In quantum wells, Thilagam has theoretically shown that the energy shifts have a different magnitude between the exciton and biexciton states. ${ }^{25}$ Such a study of QD systems would be interesting.

When an external electric field $F$ is applied, quadratic energy shifts of the exciton states $E(F)$ are observed by the Stark effect:

$$
E(F)=E_{0}-p F-\beta F^{2}
$$

where $E_{0}$ is the energy for $F=0$. In most III-V bulk semiconductors, the linear coefficient $p$ is very small. ${ }^{26-28} \mathrm{Re}-$ cently, Fu has theoretically predicted a nonzero value of $p$ due to the existence of a permanent dipole moment in spherical QDs. ${ }^{29}$ However, it is still very small, typically $\sim 1 e \cdot \AA$ in QDs of a few tens of $\mathrm{nm}$ in size. On the other hand, in the case of the S-K QDs having pyramidal or lens shapes (e.g., 
$\mathrm{Al}_{x} \mathrm{In}_{1-x} \mathrm{Ass},{ }^{17}$ InAs, ${ }^{18,21,22}$ and $\left.\mathrm{In}_{x} \mathrm{Ga}_{1-x} \mathrm{As},{ }^{5,23}\right)$, it has been clarified that there exists a large permanent dipole moment. Patanè et al. have pointed out that the localization of the electron is above the hole in $\operatorname{In}_{x} \mathrm{Ga}_{1-x} \mathrm{As}^{23}$ In other InAs QD samples, however, the confined holes are localized above the electrons in the QDs, ${ }^{18,22}$ which is opposite to that predicted by theoretical calculations. The discrepancy between the experiments and theoretical calculations is considered to be due to nonuniform indium composition in real systems. ${ }^{19}$ It is thus important to examine the existence of a permanent dipole moment and its direction in QDs of other materials. Furthermore, a permanent dipole moment in a biexciton state has never been carefully studied.

Turning our attention to the magneto-optical effects, magnetic field has historically been employed to artificially generate confined states in bulk semiconductors. In lowdimensional semiconductors, the excitonic states in a magnetic field are characterized by three energy scales: the lateral-size quantization energy, the exciton effective Rydberg energy, and the magnetic confinement energy. The magnetic length $\lambda$, which is equivalent to the cyclotron radius, is defined by

$$
\lambda=\sqrt{\frac{\hbar}{e B}},
$$

where $e$ and $B$ are the electronic charge and the magnetic field, respectively. The magnetic length characterizes the scale of magnetic confinement. We can estimate $\lambda$ to be 26 $\mathrm{nm}$ at $1 \mathrm{~T}$ and $8 \mathrm{~nm}$ at $10 \mathrm{~T}$. Since the semiconductor QDs have sizes of a few tens of nm, the magnetic length is comparable to the sizes of typical QDs in this magnetic field range. Therefore, it is interesting to investigate the competition between magnetic confinement and quantum confinement in QDs.

The exciton states show Zeeman splittings and diamagnetic shifts in a magnetic field. In many cases, these changes are much smaller than the inhomogeneous broadening of the PL band. ${ }^{30-32}$ In addition, when the lateral quantization and the Coulomb attraction are increased by decreasing the QD size, the magnetic confinement becomes less important, leading to a smaller diamagnetic shift. It is thus necessary to investigate PL from single QDs. The PL from single QDs in the magnetic field has been reported by several groups. ${ }^{1,32-37}$ Bayer et al. have observed a systematic decrease in the PL peak energy shift with decreasing size in large QDs fabricated from deep etched quantum wells, and related it to the transition from $2 \mathrm{D}$ to $0 \mathrm{D} .{ }^{38}$ On the other hand, the diamagnetic coefficient has almost a constant value in very small QDs. ${ }^{32}$ It will be interesting to study these quantities in the intermediate size regime using QDs of different sizes.

Here we present the optical response of the InP single QDs in external electric and magnetic fields. From the optical measurements we extract the quadratic Stark coefficients of excitons and biexcitons. Our results reveal the existence of permanent dipole moments in both exciton and biexciton states. Further, the size dependence of the diamagnetic coefficients is discussed.

\section{EXPERIMENTAL DETAILS}

The samples used in this study were prepared by means of metal-organic vapor phase epitaxy (MOVPE), as described in detail in Ref. 39. Self-assembled InP QDs sandwiched between two insulating $\mathrm{Ga}_{0.5} \mathrm{In}_{0.5} \mathrm{P}$ barriers of $180 \mathrm{~nm}$ thickness each were grown on a Si doped $\left(n^{+}\right)$GaAs (001) substrate. In order to apply a dc bias to the sample, an $n-i$-Schottky diode structure was fabricated by depositing a semi-transparent gold layer of $20 \mathrm{~nm}$ thickness onto the sample surface.

As the excitation light source of the optical measurements, the $488 \mathrm{~nm}$ line of a continuous wave Ar-ion laser was used. For the single dot spectroscopic study in an electric field, a confocal micro-photoluminescence ( $\mu$-PL) system was adapted. ${ }^{3}$ The samples were set on a cold-finger of a liquid He flow-type cryostat and cooled down to $4 \mathrm{~K}$. The unfocused laser beam with a diameter of $\sim 5 \mathrm{~mm}$ was irradiated on the sample surface to achieve a uniform excitation intensity. The sample PL was collected using a microscope objective lens with a numerical aperture of 0.42 . A pinhole was placed on the image plane of the microscope to select a single QD. The spatial resolution of this system is better than $2 \mu \mathrm{m}$.

In order to measure the $\mu$-PL spectra by a conventional macroscopic configuration using a superconducting split-coil magnet up to $10 \mathrm{~T}$, the QDs were confined in small mesas. ${ }^{32}$ Micro-patterns were drawn on the sample surface by means of photolithography. Most part of the sample surface was chemically etched by $\mathrm{HCl}: \mathrm{H}_{2} \mathrm{O}=2: 1$ at $30^{\circ} \mathrm{C}$ leaving behind small portions of size about $3 \times 3 \mu \mathrm{m}^{2}$. The spatial separation between the mesas is $100 \mu \mathrm{m}$. One of the mesas was selected by setting a pinhole of diameter $100 \mu \mathrm{m}$ on the sample.

In both measurements in the electric field and the magnetic field, the PL signal was analyzed using a $50 \mathrm{~cm}$ single monochromator, and then detected by a charge coupled device camera cooled by liquid nitrogen. The spectral resolution of the detection system was better than $300 \mu \mathrm{eV}$.

\section{RESULTS AND DISCUSSION}

\section{A. $\mu$-PL spectra from a single QD}

Figure 1 shows the excitation power dependence of the $\mu$-PL spectra of a single InP QD measured without applying an external field. Under very weak excitation of less than $P=1 \mathrm{~mW} / \mathrm{cm}^{2}$, a single sharp PL line denoted by $\mathrm{X}$ was observed at $\sim 1.671 \mathrm{eV}$. When the excitation power was slightly increased, a new line $\mathrm{XX}$ indicated by the arrow was observed at the lower energy side of $X$. The inset in Fig. 1 plots the $\mu$-PL intensities of the PL lines $\mathrm{X}$ and $\mathrm{XX}$ by closed and open circles, respectively.

It is instructive to analyze these results using a simple rate-equation model. The probability of the formation of an $N$-exciton state in a QD can be written as ${ }^{3}$

$$
f_{N}=\frac{\alpha^{N}}{N !} e^{-\alpha}
$$




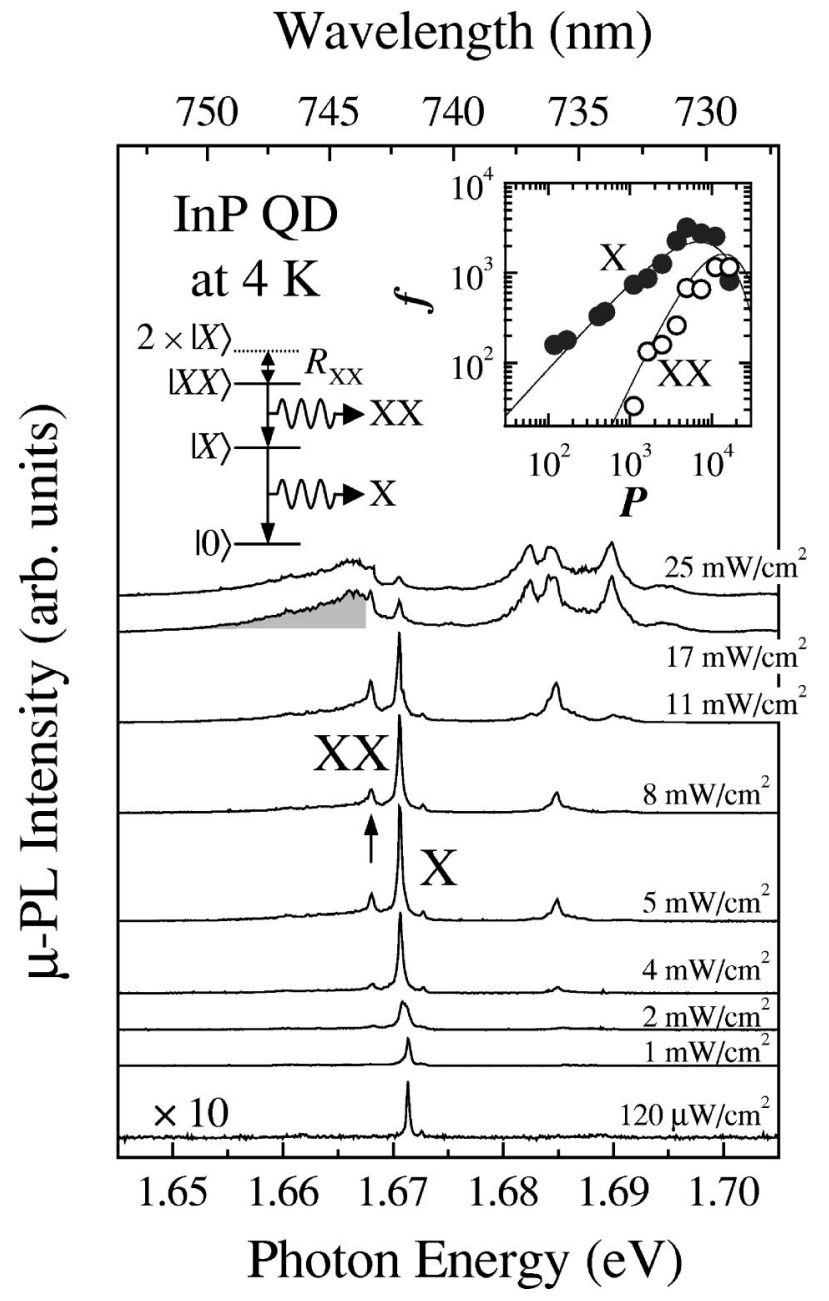

FIG. 1. Excitation power dependence of the $\mu$-PL spectra from a single InP QD without an external field. The PL peaks denoted by $\mathrm{X}$ and $\mathrm{XX}$ come from confined excitons and biexcitons, respectively. The PL from more than three excitons is observed around $1.685 \mathrm{eV}$ and below the biexciton peak (shaded area). Inset: the PL intensities $f$ of $\mathrm{X}$ and $\mathrm{XX}$ lines as a function of the excitation power density $P$ in $\mu \mathrm{W} / \mathrm{cm}^{2}$ units. The solid curves are fitted by Eq. (3).

where $\alpha$ stands for the exciton generation rate that is proportional to the excitation power $P$. As shown by the solid curves, the experimentally observed excitation power dependence of the PL lines X and XX are both well reproduced using a single parameter $\alpha$. It is thus concluded that the PL lines $\mathrm{X}$ and $\mathrm{XX}$ arise from the radiative decay of single excitons $(N=1)$ and biexcitons $(N=2)$ in their ground states, respectively, i.e., $X$ line comes from the transition of the confined exciton $|X\rangle$ to the ground state $|0\rangle$, while XX line reflects the transition from the biexciton state $|X X\rangle$ to the exciton state $|X\rangle$ (see the schematic drawn in Fig. 1). Since the exchange interaction in III-V QDs is very small, the energy separation of the PL peaks $\mathrm{X}$ and $\mathrm{XX}$ indicates the biexciton binding energy $R_{\mathrm{XX}}$. In the case of InP QDs, a typical value of the biexciton binding energy is about 3 $\mathrm{meV}^{3}$, which is a few times larger than that of bulk InP. ${ }^{40}$ When the excitation power was more than $\sim 15 \mathrm{~mW} / \mathrm{cm}^{2}$, the PL intensity of the lower-energy side shoulder of XX

\section{Wavelength (nm)}

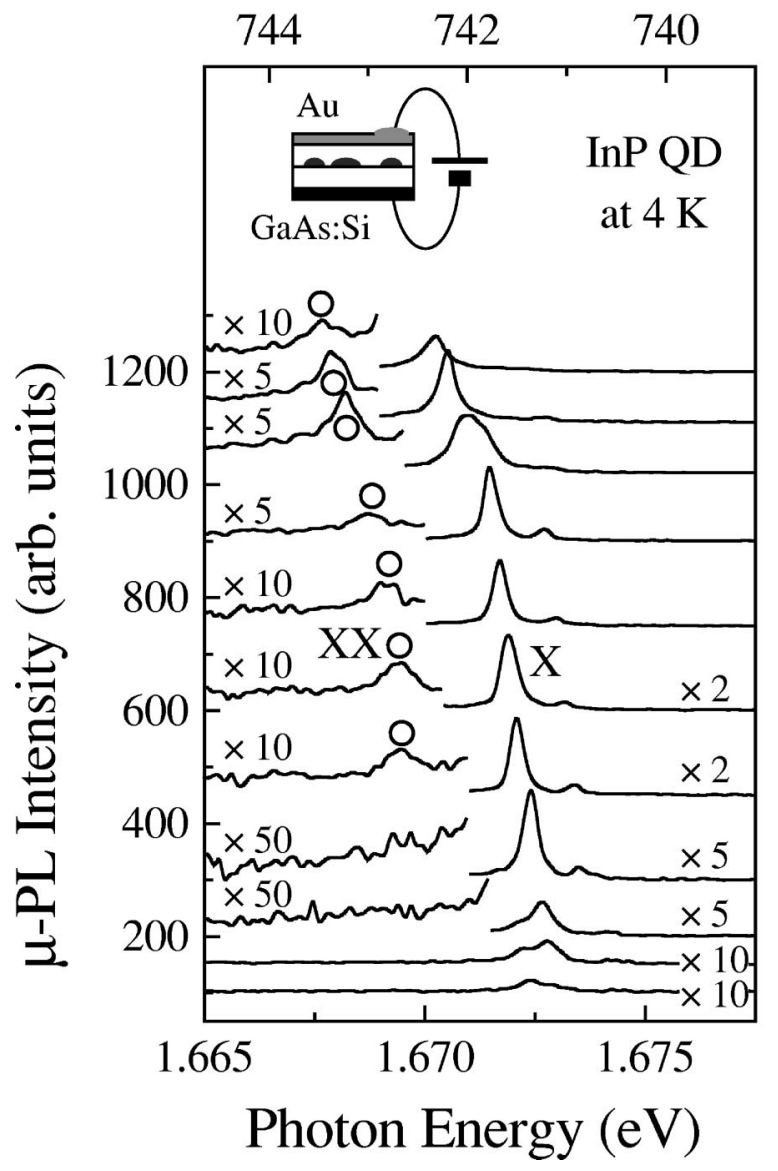

FIG. 2. Effect of an external electric field on the $\mu$-PL spectra of the QD shown in Fig. 1. The excitation power density is $8 \mathrm{~mW} / \mathrm{cm}^{2}$. The offset of each spectrum along the ordinate shows the bias applied to the sample. The applied field direction is schematically shown in the inset. With decreasing applied bias, the PL peaks from excitons $(\mathrm{X})$ and biexcitons $(\mathrm{XX})$ show blue shifts.

became strong (shaded area in Fig. 1). We consider that this shoulder originates from many exciton states, i.e., the exciton complexes composed from more than three electron-hole pairs. The PL from the confined excitons in the excited states was observed at $\sim 1.685 \mathrm{eV}$, i.e., the energy separation between the ground and first excited states is about $14 \mathrm{meV}$. First, the effect of an external electric field on the exciton and biexciton states was studied using this QD.

\section{B. Quantum confined Stark effect}

Figure 2 shows a series of the $\mu$-PL spectra of the InP QD measured by systematically changing an external electric field. In this figure, the offset of each spectrum along the vertical direction indicates the applied electric field in $\mathrm{mV}$ units. The field was taken as positive when the field lines point from the Au contact on the surface to the GaAs substrate (see the schematic shown in Fig. 2). The excitation power was kept at $8 \mathrm{~mW} / \mathrm{cm}^{2}$ throughout the experiments. The PL peak from the biexciton XX is clearly observed as denoted by the open circles, in addition to the main PL line X from the exciton. 


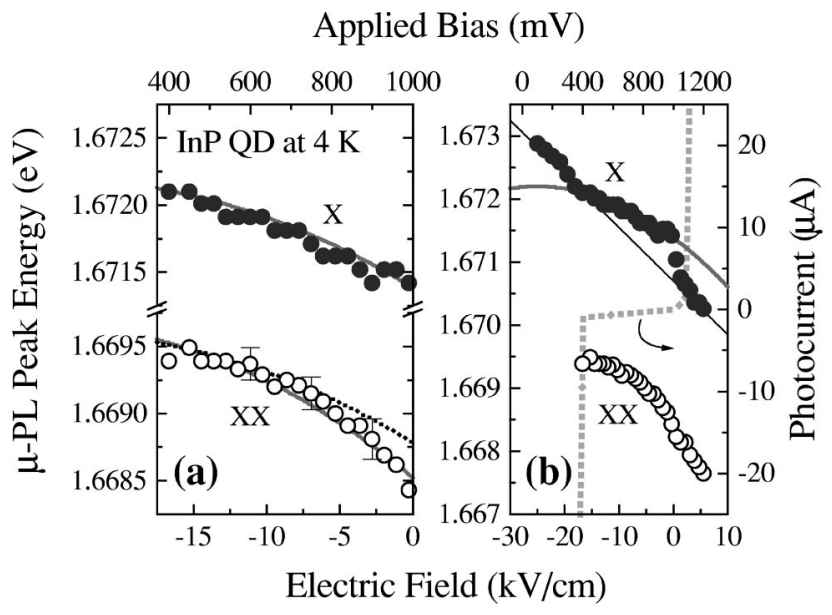

FIG. 3. PL peak energies of excitons (X, closed circles) and biexcitons (XX, open circles) as a function of applied electric field. The thick solid curves are fitted by Eq. (1). The quadratic Stark coefficient of the biexciton has a $40 \%$ larger value than that of the exciton. When the same value was assumed, a good fit was not obtained as shown by the thin dotted line in Fig. 3(a). The thick dotted curve in Fig. 3(b) is the photocurrent measured under the Ar laser irradiation of $8 \mathrm{~mW} / \mathrm{cm}^{2}$. When the photocurrent is small, the peak energy shows a quadratic shift with respect to the electric field, while it shows a linear shift (thin solid line) when the photocurrent is large. The magnification of Fig. 3(b) is shown in Fig. 3 (a), where the photocurrent is very small.

The PL spectrum measured under the open circuit situation was reproduced when the applied bias was about 1000 $\mathrm{mV}^{41}$ The distinct blue shifts of the $\mu$-PL lines from the exciton and biexciton were observed with the decrease of the applied bias. Note that when the applied bias is less than $1000 \mathrm{mV}$, the electric field through the $\mathrm{Ga}_{0.5} \mathrm{In}_{0.5} \mathrm{P}$ and $\mathrm{InP}$ layers is negative, i.e., the field lines point from the substrate to the surface. Further, the PL intensities of the excitons and biexcitons became weak as the applied bias was decreased.

Figure 3 shows the PL peak energies from the exciton X and biexciton XX shown in Fig. 2 as a function of the electric field by closed and open circles, respectively. Figure 3(b) shows the PL peak energies when the field was changed over a range wider than that in Fig. 3(a). The photocurrent $I$ measured under the Ar laser irradiation is also plotted by the thick dotted curve in Fig. 3(b). Since the photocurrent is zero when the applied bias is $\sim 1000 \mathrm{mV}$, the valence and conduction bands are flat at this point, which is consistent with the result mentioned above. The photocurrent is very small between -17 and $0 \mathrm{kV} / \mathrm{cm}(|I|<1 \mu \mathrm{A})$, while the photocurrent abruptly increases outside of this region. When the photocurrent is large, the influence of the inflow of the carriers into the QDs from the electrode and the substrate has to be taken into consideration, which causes the increase of the sample temperature (Ohmic heating) and the formation of charged excitons as briefly discussed below. We therefore discuss the energy shift of the PL peaks for electric fields between -17 and $0 \mathrm{kV} / \mathrm{cm}$ as the first step in our analysis.

Figure 3(a) shows the magnification of Fig. 3(b). As shown by the solid lines, the transition energies $E(F)$ of the exciton $\mathrm{X}$ and the biexciton $\mathrm{XX}$ are well fitted by Eq. (1). It was found that the linear coefficient $p$ has a nonzero value, which implies the existence of a nonzero permanent dipole moment in these QDs. In other words, the centers of gravity of the confined electron and hole wave functions are spatially separated along the growth direction. We find that $p$ is positive, i.e., the holes are located near the base of the QD, while the electrons are distributed above the holes.

We note that the direction of the electron-hole alignment is consistent with the recent theoretical calculations on InP QDs. ${ }^{42}$ The calculations show that holes tend to be localized closer to the base of the QD for two reasons: $(i)$ the presence of the wetting layer at the base that pulls the hole towards it and (ii) the strain profile in the barrier region above the QD that shifts the valence band edge to lower energies, pushing the hole into the base of the QD. On the other hand, the electron density is more spread out in the QD because of the lighter mass of the electrons and the dilational strain in the barrier lowering the conduction band edge in the barrier material. Similar results have also been reported for InAs QDs without indium segregation. ${ }^{15,19}$ The existence of a nonzero permanent dipole moment has been experimentally observed in exciton states of several kinds of QDs as mentioned above. However, in most InAs QD samples, the sign of the dipole moment is opposite from what we observe. It is believed that the reversal of the electron-hole spatial distribution in InAs/GaAs QDs is due to nonuniform indium distribution.

From the fittings, we evaluated the permanent dipole moment $p$ for the excitons to be $p_{\mathrm{X}}=1.0 \times 10^{-28} \mathrm{Cm}$ and that for the biexcitons to be $p_{\mathrm{XX}}=1.4 \times 10^{-28} \mathrm{Cm}$. We note that in Eq. (1), $p$ indicates the $z$ component (the growth direction) of the dipole moment

$$
p=\sum q_{i} z_{i},
$$

where $q_{\mathrm{h}}=+e$ for a hole and $q_{\mathrm{e}}=-e$ for an electron. We can therefore estimate the electron-hole spatial separation $d$ $=\left\langle\left|z_{\mathrm{e}}-z_{\mathrm{h}}\right|\right\rangle$ along the $z$ axis to be $d_{\mathrm{X}}=7 \AA$ for the excitons. ${ }^{43}$ Noting that the observed PL energy for the biexciton is the difference between the biexciton and exciton states (see the schematic drawn in Fig. 1), the dipole moment of the biexciton state is $p_{\mathrm{XX}}+p_{\mathrm{X}}=2.4 \times 10^{-28} \mathrm{Cm}$. Again, from Eq. (4), this corresponds to an average electron-hole separation of $d_{\mathrm{XX}}=8 \AA$ for the biexcitons. To the best of our knowledge, this is the first report on a nonzero permanent dipole moment in a biexciton state.

Regarding the quadratic Stark coefficient $\beta$ for the biexcitons, the fit was not good when the same value as that for the excitons is assumed [dotted curve in Fig. 3(a)]. The best fit was obtained when a $40 \%$ larger value than that for the excitons was employed [solid curve in Fig. 3(a)]. We evaluated the Stark coefficient for the excitons to be $\beta_{\mathrm{X}}=2.1$ $\times 10^{-31} \mathrm{Fcm}^{2}$ and that for the biexcitons to be $\beta_{X}=2.9$ $\times 10^{-31} \mathrm{Fcm}^{2}{ }^{44}$

When the electric field is either $F<-17 \mathrm{kV} / \mathrm{cm}$ or $F$ $>0 \mathrm{kV} / \mathrm{cm}$, a considerable amount of photocurrent flows in the sample as shown by the dotted curve in Fig. 3(b). The PL peak $X$ from the confined exciton no longer shows a quadratic energy shift, but shifts linearly with the field. The en- 


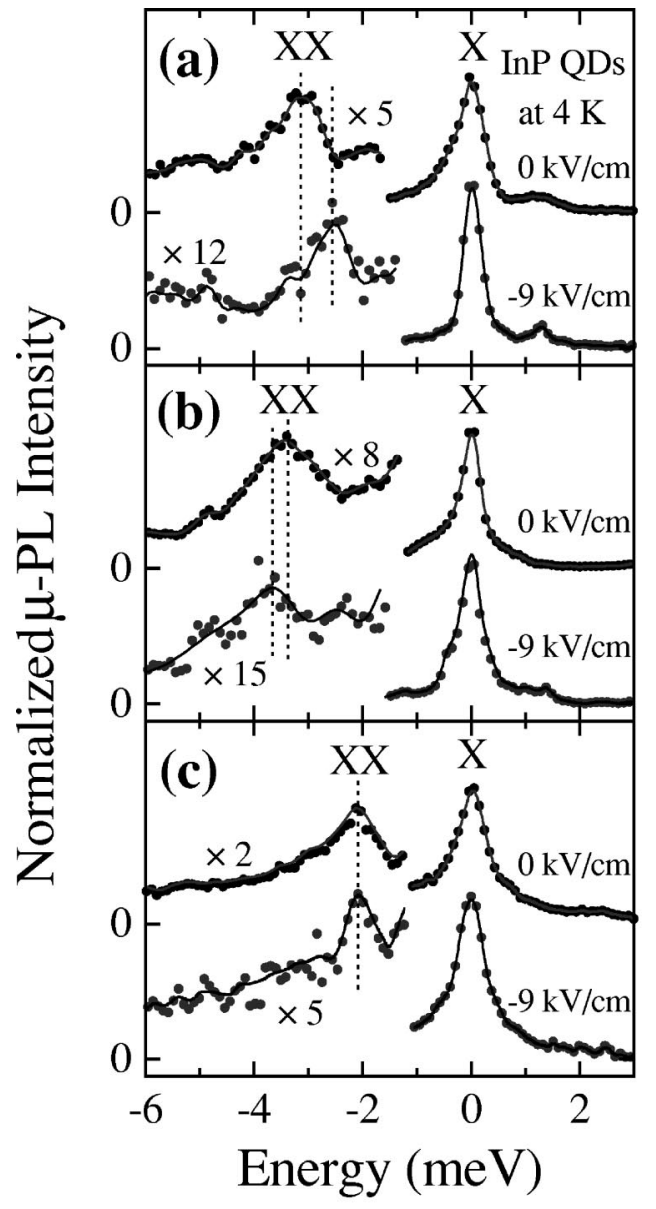

FIG. 4. $\mu$-PL spectra of three QDs with and without an applied electric field. The origins of the abscissas are taken at the PL peaks of the confined exciton lines X. The lower-energy-side peaks of the exciton lines come from the biexcitons XX. The energy separations between the exciton and biexciton lines differ from dot to dot in an electric field, reflecting the variation of the quadratic Stark coefficients. The PL peak energies of the excitons at zero electric field are (a) $1.671 \mathrm{eV}$, (b) $1.715 \mathrm{eV}$, and (c) $1.652 \mathrm{eV}$, respectively.

ergy shift becomes large on both sides of the small photocurrent region. The larger energy shift is not due to the increase of the sample temperature because an increase in the sample temperature results in a redshift of PL peak. ${ }^{45}$ However, the PL peak shifts to the higher energy side when the field is $F$ $<-17 \mathrm{kV} / \mathrm{cm}$. Instead, this large energy shift can be explained as follows: when the photocurrent is large, the probability of the trapping of the excess carriers into the QDs is enhanced, where the carriers are supplied from the electrode and the substrate. The excess carriers form coupled states with the excitons, resulting in new excitations, namely charged excitons. Unlike the excitons which are electrically neutral, the charged excitons are more sensitive to the electric field. Since the probability of the formation of the charged excitons is high when the electric field is either below $-17 \mathrm{kV} / \mathrm{cm}$ or above $0 \mathrm{kV} / \mathrm{cm}$ and since the charged excitons are considered to be sensitive to the electric field, the energy shift becomes larger than that in the small photocurrent region.

We then investigated the optical responses of the exciton

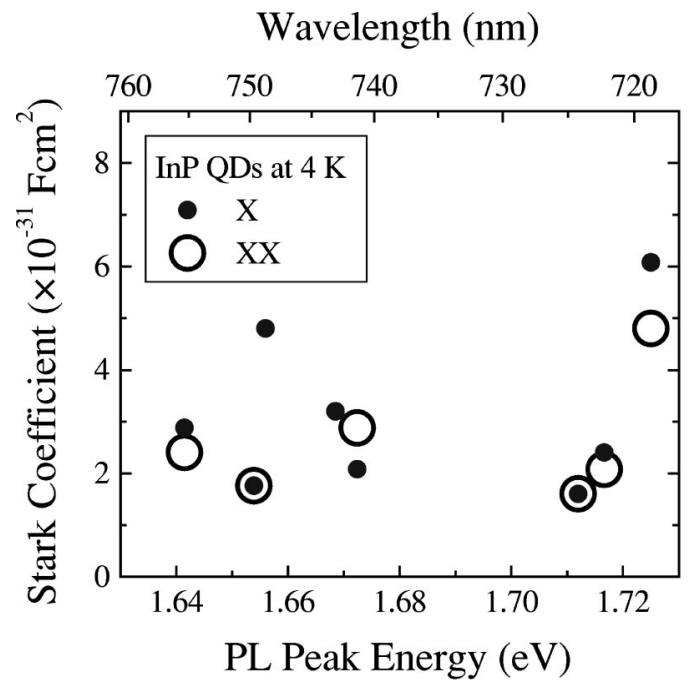

FIG. 5. Plots of quadratic Stark coefficients of excitons (X, closed circles) and biexcitons (XX, open circles) as a function of the PL peak energies at zero electric field.

and biexciton states using several QDs in the same sample. Figure 4 shows the $\mu$-PL spectra when the field is $F$ $=0 \mathrm{kV} / \mathrm{cm}$ (flat band condition) and $F=-9 \mathrm{kV} / \mathrm{cm}$. The sharp main PL peak of each spectrum shown in Fig. 4 comes from excitons and the small peak observed at the lower energy side arises from biexcitons. The abscissa shows the energy shift from the confined exciton state. The PL peak energies of the excitons are (a) $1.671 \mathrm{eV}$, (b) $1.715 \mathrm{eV}$, and (c) $1.652 \mathrm{eV}$. The spectra shown in Fig. 4(a) were measured using the QD shown in Figs. 1-3. As mentioned above, the permanent dipole moment has a nonzero value and the quadratic Stark coefficient of the biexcitons has a larger value than that of the exciton in this QD. Eventually, the energy separation between the PL peaks from the excitons and biexcitons becomes small when a negative field is applied.

The magnitude of the quadratic Stark coefficients of the excitons and biexcitons differs from dot to dot. For example, the energy separation of the PL peaks from the excitons and biexcitons becomes large in some QDs when the negative bias is applied as shown in Fig. 4(b), while the separation is almost constant in other QDs as shown in Fig. 4(c).

Figure 5 plots the quadratic Stark coefficients of the excitons and biexcitons examined in several QDs. We found that in most of the QDs $\beta$ ranges between $1 \times 10^{-31}$ and 6 $\times 10^{-31} \mathrm{Fcm}^{2}$. The PL peak energy reflects the size of QDs. We note that $\beta$ measures the polarizability of the QDs apart from a constant. In very thin quantum wells, Barker and O'Reilly have theoretically concluded that the polarizability increases with the width. ${ }^{19}$ A similar dependence may be expected in QDs. However, a systematic change with respect to the PL peak energy (i.e., the QD size) was not observed in InP QDs within the resolution of our experimental setup. The fluctuation of the values is probably due to a slight difference in shape of each QD. ${ }^{46}$

\section{Magnetic and quantum confinement}

Figure 6 shows the comparison of the excitation power dependence of the PL spectra obtained by the (a) macro- 


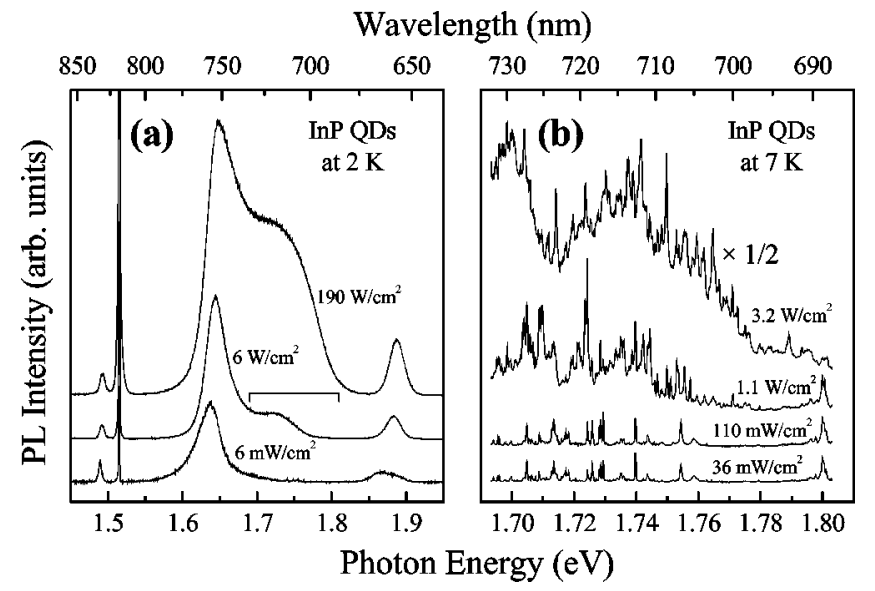

FIG. 6. Comparison of PL spectra of (a) as grown and (b) mesa samples under various excitation powers. The square bracket in Fig. 6(a) shows the energy region shown in Fig. 6(b). The state filling effect was significant when the excitation power was above $\sim 1 \mathrm{~W} / \mathrm{cm}^{2}$.

scopic and (b) microscopic methods. For the measurements, (a) the as-grown sample and (b) the QDs in a small mesa were used. The bracket of Fig. 6(a) indicates the energy region shown in Fig. 6(b). In the macroscopic observations, the state filling effects, i.e., a broadening and a high energy shift of the PL band, were not observed when the excitation power density was lower than about $1 \mathrm{~W} / \mathrm{cm}^{2}$. We note that the excitation threshold of the state filling in the sample shown in Fig. 6 is higher than that in Fig. 1, because the areal density of the QDs in Fig. 6 is high. In the $\mu$-PL spectra (b), the number and energies of the PL peaks are the same be-

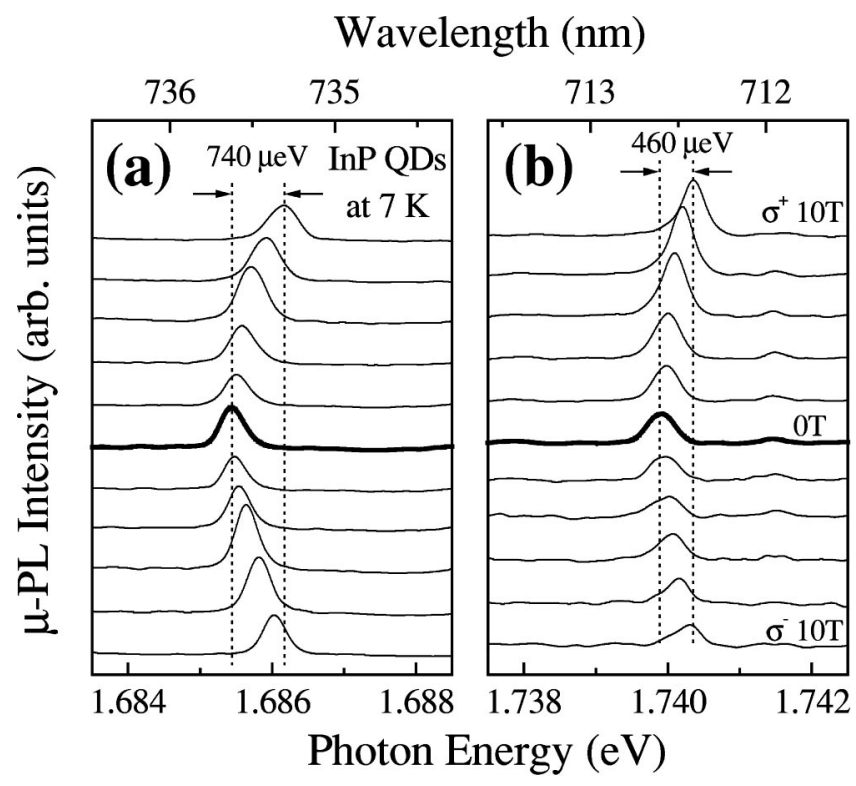

FIG. 7. Two examples of the PL energy shifts in a magnetic field. Measurements were performed in the Faraday configuration $(\boldsymbol{k}\|z\| \boldsymbol{B})$. The shifts in the magnetic field measured for the $\sigma^{+}$polarization are larger than those for the $\sigma^{-}$polarization. The PL peaks which appear at the lower energy side at zero field show a large energy shift in the magnetic field.

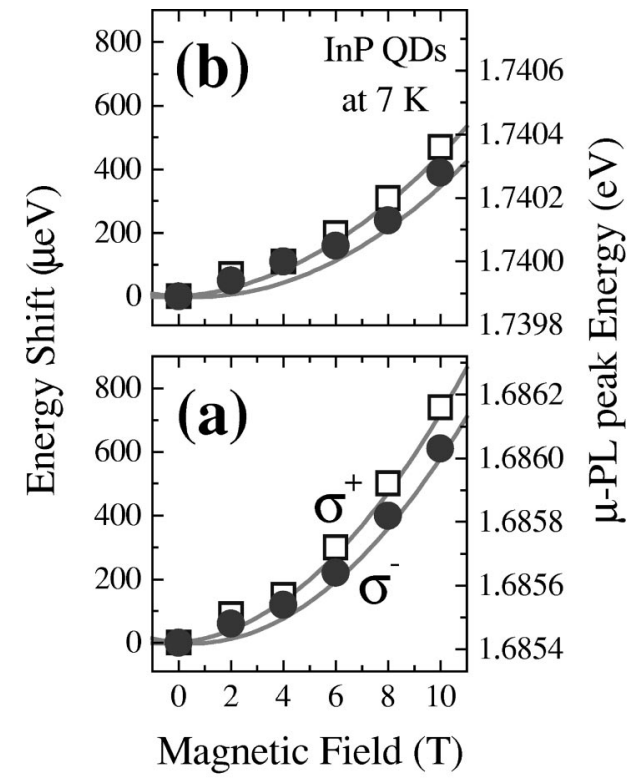

FIG. 8. Plots of the PL peaks shown in Figs. 7(a) and 7(b). The peaks denoted by the open squares were observed for $\sigma^{+}$polarization, while those denoted by the closed circles correspond to $\sigma^{-}$ polarization. Note that the maximum values of the ordinates are the same, $900 \mu \mathrm{eV}$ in both Figs. 8(a) and 8(b). The PL peak energies are well fitted by Eq. (5) as shown by the solid curves.

tween the excitation powers of 110 and $36 \mathrm{~mW} / \mathrm{cm}^{2}$. This indicates that most of the PL peaks come from the confined excitons in the ground state when the excitation power is below $\sim 100 \mathrm{~mW} / \mathrm{cm}^{2}$. The magnetic field effects on the $\mu$-PL spectra were measured under weak excitation of $100 \mathrm{~mW} / \mathrm{cm}^{2}$ as a beginning of the magnetic PL study.

Figure 7 shows two sets of the $\mu$-PL spectra from two individual QDs measured under various magnetic field strengths. The measurement was performed in the Faraday configuration $(\boldsymbol{k}\|z\| \boldsymbol{B})$ : the magnetic field $\boldsymbol{B}$ was applied normal to the sample surface, and the directions $\boldsymbol{k}$ of the photoexcitation and PL detection are parallel to the magnetic field. Here $z$ indicates the growth direction. The thick curves are the $\mu$-PL spectra at $0 \mathrm{~T}$. The upper five curves shows data for $\sigma^{+}$circular polarization, while the lower five curves correspond to $\sigma^{-}$polarization. Since the photo-excitation was made above the band gap energy of the $\mathrm{Ga}_{0.5} \mathrm{In}_{0.5} \mathrm{P}$ matrix, the polarization of the excitation laser beam caused no significant change of the $\mu$-PL spectra. The PL peaks show blueshifts on increasing the magnetic field. In all measured PL peaks, the energy shift was greater for $\sigma^{+}$than for $\sigma^{-}$.

The PL peak energies in Figs. 7(a) and 7(b) are plotted as a function of the applied magnetic field in Figs. 8(a) and 8 (b), respectively. The peaks denoted by open squares were observed for $\sigma^{+}$polarization, while those denoted by closed circles were for $\sigma^{-}$polarization. We note that the maximum values of the ordinates are the same, $900 \mu \mathrm{eV}$ in both Figs. 8(a) and 8(b). The PL peaks observed at the lower energy side show a larger energy shift with the field than those observed at the higher energy side. The PL peak energies are well fitted by 


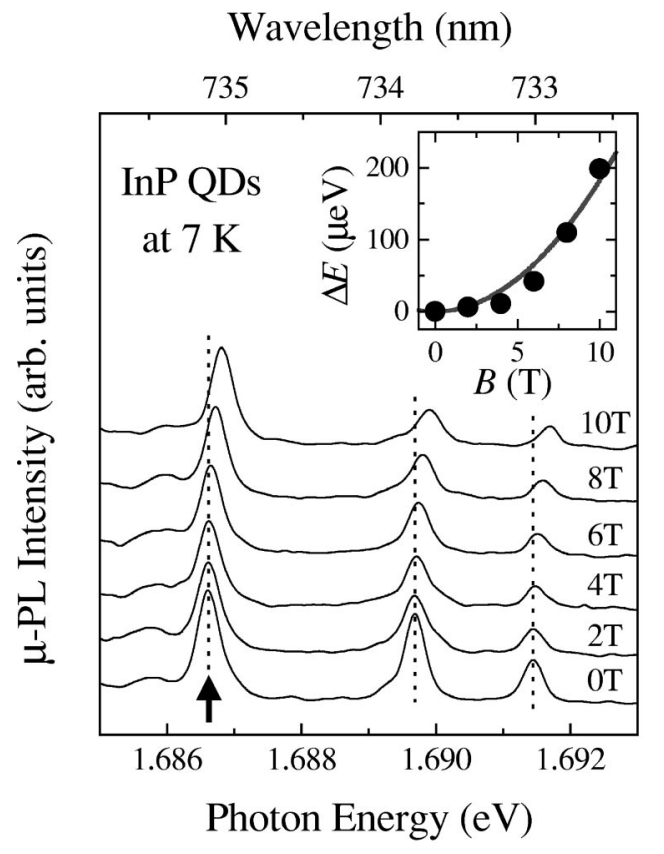

FIG. 9. $\mu$-PL spectra measured in the Voigt configuration $(\boldsymbol{k} \| z \perp \boldsymbol{B})$. The energy shift $\Delta E$ of the PL peak indicated by the arrow is shown in the inset by the closed circles as a function of the magnetic field $B$. The PL peak shows a quadratic shift as shown by the solid curve.

$$
E(B)=E_{0} \pm \frac{1}{2} \mu_{\mathrm{B}} g^{*} B+\gamma_{2} B^{2},
$$

where $E_{0}$ is the zero-field transition energy, $\mu_{\mathrm{B}}$ is the Bohr magneton, and $g^{*}$ is the effective $g$-value of the confined exciton. ${ }^{47}$ The coefficient $\gamma_{2}$ of the quadratic term in Eq. (5) is called as the diamagnetic coefficient, which is the main interest for the following part of the article.

The magnetic field effect on the $\mu$-PL spectra was also studied in the Voigt configuration $(\boldsymbol{k} \| \boldsymbol{z} \perp \boldsymbol{B})$ as shown in Fig. 9. In this measurement, a polarizer was not set in the optical path because of the weak signal. ${ }^{48}$ All PL peaks shift to the higher energy side with the increase of the magnetic field. The energy shift of the PL peak indicated by the arrow $(1.6866 \mathrm{eV}$ at $0 \mathrm{~T})$ is shown in the inset by the closed circles. Again, the PL peak energies can be well fitted using a quadratic function as shown by the solid curve.

The diamagnetic coefficients evaluated from the experimental results shown in Figs. 7-9 are summarized in Fig. 10. In the experiments, several PL peaks were examined in the Faraday (closed triangles and circles) and Voigt (open lozenges) configurations. The data denoted by the closed triangles (Faraday configuration) and open lozenges (Voigt configuration) was measured under weak excitation of $\sim 100 \mathrm{~mW} / \mathrm{cm}^{2}$, while those denoted by the closed circles (Faraday configuration) were measured under strong excitation of $\sim 300 \mathrm{~mW} / \mathrm{cm}^{2}$. Some PL peaks appear when the excitation is slightly increased. The PL peaks denoted by the closed circles were not observed under weak excitation. Therefore, these PL peaks observed under strong excitation may come from the excited states or the ground state with low PL efficiency. A large difference in the diamagnetic coefficients was, however, not observed between the PL peaks

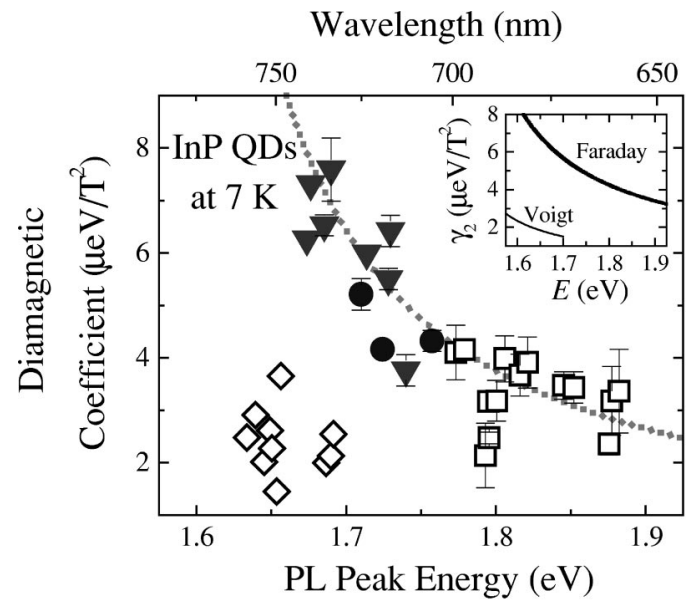

FIG. 10. Plots of the diamagnetic coefficients $\gamma_{2}$ measured in the Faraday (closed triangles and circles) and Voigt (open lozenges) configurations as a function of the PL peak energy $E$ at $0 \mathrm{~T}$. The data denoted by the closed triangles (Faraday configuration) and open lozenges (Voigt configuration) were measured under weak excitation of $\sim 100 \mathrm{~mW} / \mathrm{cm}^{2}$, while those denoted by the closed circles (Faraday configuration) were measured under strong excitation of $\sim 300 \mathrm{~mW} / \mathrm{cm}^{2}$. The open squares were measured in the Faraday configuration using QDs grown by means of GS-MBE (from Ref. 32). The dotted curve is a guide to the eyes. Inset: Plots of the model calculation using Eq. (8). The experimental results observed in the Faraday (thick curve) and Voigt (thin curve) configurations are qualitatively well reproduced.

that appear under strong and weak excitations. The open squares were measured using QDs grown by means of gassource molecular beam epitaxy (GS-MBE, for details, see Ref. 32). A systematic decrease of the diamagnetic coefficient was found with the increase of the detection energy when measured in the Faraday configuration. In other words, the diamagnetic coefficient decreases with decreasing QD size. The diamagnetic coefficient asymptotically approaches a constant value of $\sim 3 \mu \mathrm{eV} / \mathrm{T}^{2}$. This is almost the same as the value measured in the Voigt configuration. When the size of QDs becomes very small, the diamagnetic coefficient should finally approach the value of bulk $\mathrm{Ga}_{0.5} \mathrm{In}_{0.5} \mathrm{P}$ due to the leakage of the exciton wave function. ${ }^{49}$ However, the minimum value of the diamagnetic coefficient obtained in this study is $\sim 3 \mu \mathrm{eV} / \mathrm{T}^{2}$, which is much smaller than the diamagnetic coefficient in bulk $\mathrm{Ga}_{0.5} \mathrm{In}_{0.5} \mathrm{P} .{ }^{50}$ We thus consider that the small value is due to the strong confinement in small InP QDs.

In the Faraday configuration $(\boldsymbol{B} \| \boldsymbol{z})$, the magnetic fields confines the carriers in the $x-y$ plane, while they are confined in the $y-z$ plane in the Voigt configuration $(\boldsymbol{B} \| \boldsymbol{x})$. Therefore, the diamagnetic coefficient measured in the Faraday configuration reflects the wave function along the lateral direction $(x$ and $y$ ), while that measured in the Voigt configuration reflects the wave function along the growth direction $z$. Due to the flat shape of the QD, the quantum confinement along the $z$ direction is stronger than that along the $x-y$ direction, and thus the exciton wave function is already shrunk significantly along the $z$ direction even at $0 \mathrm{~T}$. In this case, an additional confinement by the magnetic field is less effective. This ex- 
plains why the diamagnetic coefficient is smaller when observed in the Voigt configuration. ${ }^{51}$

The diamagnetic coefficient can be written as

$$
\gamma_{2}=\frac{e^{2}\left\langle\rho^{2}\right\rangle}{8 \mu},
$$

where $e, \sqrt{\left\langle\rho^{2}\right\rangle}$, and $\mu$ are the electronic charge, the effective exciton size, and the reduced mass, respectively. We note that the wave functions of the electrons and holes are assumed to have the same size. The effective exciton size due to the magnetic confinement can be estimated using this relationship.

The size dependence of the diamagnetic coefficient in the Faraday configuration is qualitatively understood as follows. In a strong confinement regime, the confined exciton energy $E$ and the QD radius $R$ have the relation

$$
E=E_{\mathrm{B}}+\frac{\hbar^{2} \pi^{2}}{2 \mu R^{2}},
$$

where $E_{\mathrm{B}}$ is the band gap energy of the bulk $\operatorname{InP}\left(E_{\mathrm{B}}\right.$ $=1.42 \mathrm{eV}$ at liquid helium temperature ${ }^{52}$ ) and the second term is the confinement energy. In Eq. (7), the shape of the QD is assumed to be spherical for simplicity. The effective exciton radius is considered to be comparable with the QD size. Thus, we finally obtain the relation

$$
\gamma_{2} \simeq \frac{a}{E-E_{\mathrm{B}}}
$$

with $a=(\hbar e \pi / 4 \mu)^{2}$. In the case of InP, $a=1.7$ $\times 10^{-6} \mathrm{eV}^{2} / \mathrm{T}^{2}$ is obtained when we use $m_{\mathrm{hh}}=0.56 m_{0}\left(m_{0}\right.$ is the free electron mass) for the heavy hole and $m_{\mathrm{e}}$ $=0.08 m_{0}$ for the conduction electron to estimate the reduced exciton mass, ${ }^{52}$

$$
\mu=\left(\frac{1}{m_{\mathrm{hh}}}+\frac{1}{m_{\mathrm{e}}}\right)^{-1} .
$$

Despite the simplicity of this model, it explains our experimental results in the Faraday configuration very well, as shown by the thick curve in the inset in Fig. 10. The diamagnetic coefficient $\gamma_{2}$ gradually decreases as the observation energy $E$ increases, which is consistent with the experiment.
Further, since the height of the QDs is about a quarter of the base diameter, ${ }^{8,39}$ the diamagnetic coefficient when measured in the Voigt configuration is expected to be four times smaller than that in the Faraday configuration. Based on this model, the calculated result of the diamagnetic coefficient in the Voigt configuration is also shown by the thin curve in the inset in Fig. 10. Again, the agreement between the experiment and the model is fairly good. Therefore, we conclude that the gradual reduction of the diamagnetic coefficient with the QD size reflects the decrease of the effective exciton size due to the strong quantum confinement in the QDs.

\section{SUMMARY}

We have studied the optical properties of the InP QDs in the presence of external electric and magnetic fields. The quantum confined Stark shifts of both the exciton and biexciton states were clearly observed. The quadratic Stark coefficient was evaluated to be of the order of $10^{-31} \mathrm{Fcm}^{2}$, and it varies slightly from dot to dot. It was found that the energy separation of the PL peaks between the exciton and biexciton states changes with the applied bias, reflecting a small difference of the Stark coefficient of these states. We find that the exciton and biexciton states have a nonzero permanent dipole moment. The average spatial separation of the electron and hole wave functions along the growth axis was evaluated to be about $7 \AA$ for excitons and $8 \AA$ for biexcitons. We also found that the holes are located closer to the base of the QDs compared to the electrons. The experimental results are consistent with reported theoretical calculations.

Zeeman splitting and diamagnetic shift of the exciton state were also clearly observed by the magnetic PL measurements of single InP QDs. We found that there is a systematic decrease of the diamagnetic coefficient with decreasing size of the QDs. The diamagnetic coefficient measured in the Voigt configuration was small compared to that measured in the Faraday configuration. We concluded that the gradual reduction of the diamagnetic coefficient with the QD size reflects the decrease of the effective exciton size due to the quantum confinement.

\section{ACKNOWLEDGMENTS}

The authors thank Professor H. E. Ruda of the University of Toronto for his encouragement to summarize this work. Close collaboration with Dr. J.-S. Lee is also acknowledged.
*Corresponding author; Email: mitsuru.sugisaki@utoronto.ca Present address: Department of Physics, Graduate School of Science, Osaka City University, 3-3-138 Sugimoto, Sumiyoshi-ku, Osaka 558-8585, Japan.

${ }^{1}$ A. Kuther, M. Bayer, A. Forchel, A. Gorbunov, V.B. Timofeev, F. Schäfer, and J.P. Reithmajer, Phys. Rev. B 58, 7508 (1998).

${ }^{2}$ F. Findeis, A. Zrenner, G. Böhm, and G. Abstreiter, Phys. Rev. B 61, R10579 (2000).

${ }^{3}$ M. Sugisaki, H.-W. Ren, S.V. Nair, K. Nishi, and Y. Masumoto, Solid State Commun. 117, 435 (2001).

${ }^{4}$ A. Hartmann, Y. Ducommun, E. Kapon, U. Hohenester, and E. Molinari, Phys. Rev. Lett. 84, 5648 (2000).
${ }^{5}$ F. Findeis, M. Baier, A. Zrenner, M. Bichler, G. Abstreiter, U. Hohenester, and E. Molinari, Phys. Rev. B 63, 121309(R) (2001); M. Baier, F. Findeis, A. Zrenner, M. Bichler, and G. Abstreiter, ibid. 64, 195326 (2001).

${ }^{6}$ D. Gammon, E.S. Snow, B.V. Shanabrook, D.S. Katzer, and D. Park, Science 273, 87 (1996).

${ }^{7}$ T. Kümmell, R. Weigand, G. Bacher, A. Forchel, K. Leonardi, D. Hommel, and H. Selke, Appl. Phys. Lett. 73, 3105 (1998).

${ }^{8}$ M. Sugisaki, H.-W. Ren, S.V. Nair, K. Nishi, S. Sugou, T. Okuno, and Y. Masumoto, Phys. Rev. B 59, R5300 (1999); M. Sugisaki, H.-W. Ren, K. Nishi, and Y. Masumoto, Solid State Commun. 117, 679 (2001). 
${ }^{9}$ As a review article, S.A. Empedocles, R. Neuhauser, K. Shimizu, and M.G. Bawendi, Adv. Mater. 11, 1243 (1999).

${ }^{10}$ M. Sugisaki, H.-W. Ren, K. Nishi, and Y. Masumoto, Phys. Rev. Lett. 86, 4883 (2001), and references therein.

${ }^{11}$ P. Michler, A. Kiraz, C. Becher, W.V. Schoenfeld, P.M. Petroff, L. Zhang, E. Hu, and A. Imamoğlu, Science 290, 2282 (2000).

${ }^{12}$ C. Santori, M. Pelton, G. Solomon, Y. Dale, and Y. Yamamoto, Phys. Rev. Lett. 86, 1502 (2001).

${ }^{13}$ V. Zwiller, H. Blom, P. Jonsson, T. Tsegaye, E. Goobar, M.-E. Pistol, L. Samuelson, and G. Björk, Appl. Phys. Lett. 78, 2476 (2001).

${ }^{14}$ H. Gotoh and H. Ando, J. Appl. Phys. 82, 1667 (1997).

${ }^{15}$ K. Chang and J.-B. Xia, Solid State Commun. 104, 351 (1997).

${ }^{16}$ W. Heller, U. Bockelmann, and G. Abstreiter, Phys. Rev. B 57, 6270 (1998).

${ }^{17}$ S. Raymond, J.P. Raynolds, J.L. Merz, S. Fafard, Y. Feng, and S. Charbonneau, Phys. Rev. B 58, R13415 (1998).

${ }^{18}$ P.W. Fry, I.E. Itskevich, D.J. Mowbray, M.S. Skolnick, J.J. Finley, J.A. Barker, E.P. O'Reilly, L.R. Wilson, I.A. Larkin, P.A. Maksym, M. Hopkinson, M. Al-Khafaji, J.P.R. David, A.G. Cullis, G. Hill, and J.C. Clark, Phys. Rev. Lett. 84, 733 (2000); P.W. Fry, I.E. Itskevich, S.R. Parnell, J.J. Finley, L.R. Wilson, K.L. Schumacher, D.J. Mowbray, M.S. Skolnick, M. Al-Khafaji, A.G. Cullis, M. Hopkinson, J.C. Clark, and G. Hill, Phys. Rev. B 62, 16784 (2000).

${ }^{19}$ J.A. Barker and E.P. O’Reilly, Phys. Rev. B 61, 13840 (2000).

${ }^{20}$ H. Gotoh, H. Kamada, H. Ando, and J. Temmyo Appl. Phys. Lett. 76, 867 (2000).

${ }^{21}$ I.E. Itskevich, S.I. Rybchenko, I.I. Tarkakovskii, S.T. Stoddart, A. levin, P.C. Main, L. Eaves, M. Henini, and S. Parnell, Appl. Phys. Lett. 76, 3932 (2000).

${ }^{22}$ W.-H. Chang, T.M. Hsu, C.C. Huang, N.T. Yeh, and J.-I. Chyi, Phys. Status Solidi B 224, 89 (2001); T.M. Hsu, W.-H. Chang, C.C. Huang, N.T. Yeh, and J.-I. Chyi, Appl. Phys. Lett. 78, 1760 (2001).

${ }^{23}$ A. Patanè, A. Levin, A. Polimeni, F. Schindler, P.C. Main, L. Eaves, and M. Henini, Appl. Phys. Lett. 77, 2979 (2000).

${ }^{24}$ D. Hessman, J. Persson, M.-E. Pistol, C. Pryor, and L. Samuelson, Phys. Rev. B 64, 233308 (2001).

${ }^{25}$ A. Thilagam, Phys. Rev. B 56, 4665 (1997).

${ }^{26}$ D.A.B. Miller, D.S. Chemla, T.C. Damen, A.C. Gossard, W. Wiegmann, T.H. Wood, and C.A. Burrus, Phys. Rev. Lett. 53, 2173 (1984).

${ }^{27}$ I. Bar-Joseph, C. Klingshirn, D.A.B. Miller, D.S. Chemia, U. Koren, and B.I. Miller, Appl. Phys. Lett. 50, 1010 (1987).

${ }^{28}$ X.B. Mei, W.G. Bi, C.W. Tu, L.J. Chou, and K.C. Hsieh, J. Vac. Sci. Technol. B 14, 2327 (1996).

${ }^{29}$ H. Fu, Phys. Rev. B 65, 045320 (2002).

${ }^{30}$ S. Nomura, L. Samuelson, M.-E. Pistol, K. Uchida, N. Miura, T. Sugano, and Y. Aoyagi, Appl. Phys. Lett. 71, 2316 (1997); S. Nomura, L. Samuelson, C. Pryor, M.-E. Pistol, M. Stopa, K. Uchida, N. Miura, T. Sugano, and Y. Aoyagi, Phys. Rev. B 58, 6744 (1998).

${ }^{31}$ B. Kowalski, S. Nomura, C. Pryor, Y. Aoyagi, N. Carlsson, M.-E. Pistol, P. Omling, L. Samuelson, and W. Seifert, Phys. Rev. B 58, 2026 (1998).

${ }^{32}$ M. Sugisaki, H.-W. Ren, K. Nishi, S. Sugou, T. Okuno, and Y. Masumoto, Physica B 256-258, 169 (1998).

${ }^{33}$ S.W. Brown, T.A. Kennedy, D. Gammon, and E.S. Snow, Phys.
Rev. B 54, R17339 (1996); D. Gammon, A.L. Efros, T.A. Kennedy, M. Rosen, D.S. Katzer, D. Park, S.W. Brown, V.L. Korenev, and I.A. Merkulov, Phys. Rev. Lett. 86, 5176 (2001).

${ }^{34}$ Y. Toda, S. Shinomori, K. Suzuki, and Y. Arakawa, Appl. Phys. Lett. 73, 517 (1998); Y. Toda, S. Shinomori, K. Suzuki, and Y. Arakawa, Phys. Rev. B 58, R10147 (1998).

${ }^{35}$ M. Bayer, A. Kuther, F. Schäfer, J.P. Reithmaier, and A. Forchel, Phys. Rev. B 60, R8481 (1999).

${ }^{36}$ M. Bayer, O. Stern, A. Kuther, and A. Forchel, Phys. Rev. B 61, 7273 (2000).

${ }^{37}$ I.E. Itskevich, S.T. Stoddart, S.I. Rybchenko, I.I. Tarkakovskii, L. Eaves, P.C. Main, M. Henini, and S. Parnell, Phys. Status Solidi A 178, 307 (2000).

${ }^{38}$ M. Bayer, S.N. Walck, T.L. Reinecke, and A. Forchel, Phys. Rev. B 57, 6584 (1998).

${ }^{39}$ H.-W. Ren, M. Sugisaki, J.-S. Lee, S. Sugou, and Y. Masumoto, Jpn. J. Appl. Phys., Part 1 38, 507 (1999).

${ }^{40}$ S. Charbonneau, L.B. Allard, A.P. Roth, and R.T. Sudersena, Phys. Rev. B 47, 13918 (1993).

${ }^{41}$ The height of the Schottky barrier is consistent with the former report: S.D. Kwon, O.K. Kwon, B.-D. Choe, H. Lim, and J.Y. Lee, J. Appl. Phys. 78, 2482 (1995).

${ }^{42}$ S.V. Nair, J. Shumway, and A. Zunger, Proceedings of the APS March meeting (Seattle, 2001).

${ }^{43}$ It has been pointed out that the electron and hole are spatially separated in multi-stacked InP QDs; In this case, the center of the wave function of the hole exists outside of the QDs: M. Hayne, R. Provoost, M.K. Zundel, Y.M. Manz, K. Eberl, and V.V. Moshchalkov, Phys. Rev. B 62, 10324 (2000); M. Hayne, M. Maes, V.V. Moshchalkov, Y.M. Manz, O.G. Schmidt, and K. Eberl, Appl. Phys. Lett. 79, 45 (2001); Such a large separation was, however, not observed in the single-layered InP QDs used in this study. The separation is much smaller than the QD size.

${ }^{44}$ Besombes et al. have recently observed the Stark shift of the neutral and charged exciton states in CdTe QDs. They found that each state has different value of the Stark coefficient: L. Besombes, K. Kheng, L. Marsal, and H. Mariette, Phys. Rev. B 65, 121314(R) (2002).

${ }^{45}$ M. Sugisaki, H.-W. Ren, S.V. Nair, J.-S. Lee, S. Sugou, T. Okuno, and Y. Masumoto, J. Lumin. 87-89, 40 (2000).

${ }^{46}$ It should be mentioned that we have measured the Stark shift under both weak (less than 0.1 excitons/dot) and strong (about 1 exciton/dot) excitation conditions to examine the effect from excess carriers on both inside and outside of the QDs. However, a significant difference was not observed in the Stark coefficients.

${ }^{47}$ We have measured several samples and found that the Zeeman splitting $\Delta E_{ \pm}=\mu_{\mathrm{B}} g^{*} B$, which reflects the effective $g$-value, is scattered over a wide range from 200 to $800 \mu \mathrm{eV}$ at $10 \mathrm{~T}$. A systematic change of the Zeeman splitting was not observed, which is the same as the case of the InAs QDs in the (Al) GaAs matrix. ${ }^{34}$ More details of the Zeeman splitting will be discussed elsewhere.

${ }^{48}$ Bayer et al. has reported a fine splitting in the Voigt configuration in $\mathrm{In}_{x} \mathrm{Ga}_{1-x}$ As QDs, where the splitting is much smaller than that observed in the Faraday configuration. ${ }^{36}$ In our case, however, such a splitting was not observed probably because of the small $g$-value in InP QDs. 
${ }^{49}$ K.L. Janssens, F.M. Peeters, and V.A. Schweigert, Phys. Rev. B 63, 205311 (2001).

${ }^{50}$ The diamagnetic coefficient of bulk $\mathrm{Ga}_{0.5} \mathrm{In}_{0.5} \mathrm{P}$ strongly depends on the direction of the magnetic field due to the formation of the $\mathrm{Cu}-\mathrm{Pt}_{\mathrm{B}}$ ordered domains. The value also depends on the degree of ordering. When the field is applied along the (100) direction, the diamagnetic coefficient is $\sim 10 \mu \mathrm{eV} / \mathrm{T}^{2}$, which is much larger than the value in the InP QDs studied here: P. Ernst, Y. Zhang, F.A.J.M. Driessen, A. Mascarenhas, E.D. Jones, C. Geng, F. Scholz, and H. Schweizer, J. Appl. Phys. 81, 2814 (1997); U. Kops, P.G. Blome, M. Wenderoth, R.G. Ulbrich, C. Geng, and F. Scholz, Phys. Rev. B 61, 1992 (2000).
${ }^{51}$ We note that the small energy shift in the Voigt configuration has also been reported in GaAs/AlAs quasi-0D excitons and macroscopically in InAlAs/AlGaAs S-K QDs: A. Zrenner, L.V. Butov, M. Hagn, G. Abstreiter, B. Böhm, and G. Weimann, Phys. Rev. Lett. 72, 3382 (1994); P.D. Wang, J.L. Merz, S. Fafard, R. Leon, D. Leonard, G. Medeiros-Ribeiro, M. Oestreich, P.M. Petroff, K. Uchida, N. Miura, H. Akiyama, and H. Sakaki, Phys. Rev. B 53, 16458 (1996).

${ }^{52}$ Landolt-Börnstein, Numerical Data and Functional Relationships in Science and Technology, edited by O. Madelung, M. Schluz, and H. Weiss, New Series, Vol. 17a (Springer-Verlag, Berlin, 1982). 